YITP-SB-13-3

\title{
On the developments of Sklyanin's quantum separation of variables for integrable quantum field theories.
}

\author{
G. NICCOLI \\ C.N. YANG INSTITUTE FOR THEORETICAL PHYSICS, STONY BROOK UNIVERSITY, \\ STONY BROOK, NY 11794-3840, USA, E-mail: niccoli@max2.physics.sunysb.edu
}

\begin{abstract}
We present a microscopic approach in the framework of Sklyanin's quantum separation of variables (SOV) for the exact solution of 1+1-dimensional quantum field theories by integrable lattice regularizations. Sklyanin's SOV is the natural quantum analogue of the classical method of separation of variables and it allows a more symmetric description of classical and quantum integrability w.r.t. traditional Bethe ansatz methods. Moreover, it has the advantage to be applicable to a more general class of models for which its implementation gives a characterization of the spectrum complete by construction. Our aim is to introduce a method in this framework which allows at once to derive the spectrum (eigenvalues and eigenvectors) and the dynamics (time dependent correlation functions) of integrable quantum field theories (IQFTs). This approach is presented for a paradigmatic example of relativistic IQFT, the sine-Gordon model.
\end{abstract}

Keywords: integrable quantum models, quantum inverse scattering method, Sklyanin's quantum separation of variables.

\section{Introduction}

The solution of quantum field theories by the complete characterization of their spectrum (eigenvalues and eigenstates) and dynamics (time dependent correlation functions) is one of the fundamental issues in mathematical physics as it should lead to exact (non-perturbative) results in several areas of physics where these models play a central role. The 1+1-dimensional case $^{1}$ is the most natural framework where to try to solve exactly this problem thanks to the powerful tools of quantum integrability ${ }^{2,3}$. Despite significant progresses obtained in the last forty years for some lattice models (like the Heisenberg spin chains), the full solution of more general integrable quantum field theories (IQFTs) is still a fundamental open problem. Our main aim is to define a microscopic approach for the exact solution of $1+1$-dimensional quantum field theories by integrable lattice regularizations in the framework of the quantum inverse scattering method (QISM). In this framework, the quantum integrable structure:

$$
\mathrm{T}(\lambda) \in \operatorname{End}(\mathcal{H}):[\mathrm{T}(\lambda), \mathrm{T}(\mu)]=0 \quad \forall \lambda, \mu \in \mathbb{C}, \quad H \in \mathrm{T}(\lambda)
$$

of a quantum model of Hamiltonian $H \in \operatorname{End}(\mathcal{H})$ on the quantum (Hilbert) space 
$\mathcal{H}$ is defined by the one-parameter family of transfer matrices:

$$
\mathrm{T}(\lambda) \equiv \operatorname{tr}_{\mathbb{C}^{2}} \mathrm{M}_{0}(\lambda), \quad \mathrm{M}_{0}(\lambda) \equiv\left(\begin{array}{c}
\mathrm{A}(\lambda) \mathrm{B}(\lambda) \\
\mathrm{C}(\lambda) \mathrm{D}(\lambda)
\end{array}\right)_{0} \in \operatorname{End}\left(\mathcal{H} \otimes \mathbb{C}^{2}\right) .
$$

Here, we are restricting ourselves to a monodromy matrix $\mathrm{M}(\lambda) \in \operatorname{End}\left(\mathcal{H} \otimes \mathbb{C}^{n}\right)$ with $n=2$; this is a solution of the so-called Yang-Baxter equation in $\operatorname{End}\left(\mathcal{H} \otimes \mathbb{C}^{2} \otimes \mathbb{C}^{2}\right)$ :

$$
R_{0,0^{\prime}}(\lambda / \mu)\left(\mathrm{M}_{0}(\lambda) \otimes 1_{0^{\prime}}\right)\left(1_{0} \otimes \mathrm{M}_{0^{\prime}}(\mu)\right)=\left(1_{0} \otimes \mathrm{M}_{0^{\prime}}(\mu)\right)\left(\mathrm{M}_{0}(\lambda) \otimes 1_{0^{\prime}}\right) R_{0,0^{\prime}}(\lambda / \mu),
$$

and $R_{0,0^{\prime}}(\lambda) \in \operatorname{End}\left(\mathbb{C}^{2} \otimes \mathbb{C}^{2}\right)$ is a solution of the Yang-Baxter equation in End $\left(\mathbb{C}^{2} \otimes\right.$ $\left.\mathbb{C}^{2} \otimes \mathbb{C}^{2}\right)$. The elements of $\mathrm{M}(\lambda)$ are the generators of a representation in $\mathcal{H}$ of the Yang-Baxter algebra and, for invertible $R$-matrix, the commutation relations (3) imply the mutual commutativity of the one-parameter family of transfer matrices $\mathrm{T}(\lambda)$.

\subsection{Local fields identification problem in S-matrix formulation}

It is worth recalling that some classes of massive integrable quantum field theories (IQFTs) in infinite volume can be defined avoiding a microscopic lattice regularization. Indeed, they admit an on-shell ${ }^{4}$ exact and complete characterization by the exact S-matrices which fixes the asymptotic particle dynamics. The main difficulty here is that any information needs to be extracted from the particle dynamics. In particular, a direct connection between local fields and form factors (their matrix elements on asymptotic particle states) is absent and the form factors are characterized axiomatically as solutions of a set of functional equations ${ }^{5}$ completely fixed by the exact S-matrix. Different methods have addressed this longstanding problem and the description of massive IQFTs as (superrenormalizable) perturbations of conformal field theories by relevant local fields ${ }^{6}$ has characterized one important research line. The consequent hypothesis of isomorphism of the local field content between massive theories and the corresponding ultraviolet conformal ones has been verified for some fundamental IQFTs both for the chiral ${ }^{7}$ and the non-chiral local fields ${ }^{8}$ by form factor analysis. These are important results on the global structure of the local operator spaces of the massive IQFTs but they do not really lead to the identification of particular local fields. It is worth recalling that in ${ }^{9}$ a criterion based on the quasi-classical characterization of the local fields has been introduce to define the correspondence between local fields and form factors. It has been fully described in the special cases of the restricted sine-Gordon model at the reflectionless points for chiral fields and verified on the basis of counting arguments. From the above discussion it is then clear that in the S-matrix formulation the main open problem remains the absence of a direct reconstruction of the quantum local fields.

${ }^{a}$ It is worth mentioning that the new fermionic structures described in ${ }^{10}$, appearing from the lattice regularization given in terms of the XXZ spin-1/2 quantum chain, have been used recently to investigate the structure of form factors of the sine-Gordon model in the infinite volume limit. Remarkably the authors of ${ }^{11}$ were able to reproduce the results of the papers ${ }^{9}$ from this different approach. 


\subsection{Integrable microscopic approach in SOV framework}

One of our main motivations to develop an integrable microscopic approach to quantum field theories is to introduce an exact setup where to overcome the identification problem in the S-matrix formulation. Indeed, in the QISM framework, we can use the so-called solution of the quantum inverse scattering problem, a central achievements in the Lyon group method $^{12}$, which applies to a large class of lattice integrable quantum models and allows to write explicitly the local operators in terms of the global Yang-Baxter generators. Such a result plays a key role in the derivation of multiple integral representations of correlation functions as it is at the basis of the algebraic computations of the local operator actions on the transfer matrix eigenstates. The Lyon group method has been develop by using the algebraic Bethe ansatz (ABA) as central tool for the spectrum characterization. However, ABA does not work for important integrable quantum models on the contrary of the Sklyanin's quantum separation of variables $(\mathrm{SOV})^{13}$. This beautiful method is quite general and powerful to describe the spectrum of these models; it leads to both the eigenvalues and the eigenstates of the transfer matrix with a spectrum construction (which under simple conditions) has as built-in feature its completeness. Moreover, in the SOV framework, for the so far analyzed quantum models ${ }^{14-16}$ it was an easy task to prove their complete quantum integrability; i.e. the simplicity of the transfer matrix spectrum. Our aim is to develop a method based on the Sklyanin's SOV which exactly characterize the spectrum and the dynamics (correlation functions) of IQFTs according to the following general schema:

A) Solution of the spectral problem, for the lattice and the continuum theories: A1) Solution of the spectral problem for the integrable lattice regularizations; i.e. SOV construction of transfer matrix eigenstates and eigenvalues. A2) Reformulation of the spectrum in terms of nonlinear integral equations (of thermodynamical Bethe ansatz type) and definition of finite volume quantum field theories by continuum limit. A3) Derivation of S-matrix description of the spectrum in the IR limit, infinite volume. A4) Derivation of the renormalization group fixed point conformal spectrum in the UV limit.

B) Exact formulae for the correlation functions: B1) Reconstruction of the local operators in terms of the Sklyanin's quantum separate variables. B2) Determinant form for the scalar product of the class of separate states, which contains also the transfer matrix eigenstates. B3) Matrix elements of local operators on transfer matrix eigenstates. B4) Thermodynamical limit and derivation of multiple integral formulae for correlation functions.

\subsection{On the Sklyanin's quantum separation of variables}

Following Sklyanin ${ }^{13}$, we would like first to present a possible definition of quantum separate variables for an integrable quantum model. Let $Y_{n}, P_{n} \in \operatorname{End}(\mathcal{H})$ be $\mathrm{N}$ couples of canonical conjugate operators $\left[Y_{n}, Y_{m}\right]=\left[P_{n}, P_{m}\right]=0,\left[Y_{n}, P_{m}\right]=$ 
$\delta_{n, m} / 2 \pi i$ and let us assume that $\left\{Y_{1}, \ldots, Y_{\mathrm{N}}\right\}$ are simultaneously diagonalizable operators with simple spectrum on $\mathcal{H}$. Then, we can present the following:

Definition The set of operators $\left\{Y_{1}, \ldots, Y_{\mathrm{N}}\right\}$ define the quantum separate variables for the spectral problem of the one parameter family of conserved charges $\mathrm{T}(\lambda)$ if and only if there exist (under an appropriate definition of the operator order) $\mathrm{N}$ quantum separate relations of the type:

$$
F_{n}\left(Y_{n}, P_{n}, \mathrm{~T}\left(Y_{n}\right)\right)=0 \quad \forall n \in\{1, . ., \mathrm{N}\} .
$$

These are quantum analogues of the classical separate relations in the HamiltonJacobi's approach and are here used to solve the spectral problem of $\mathrm{T}(\lambda)$. Thanks to (4), in the eigenbasis of $\mathcal{H}$ formed out of $\left\{Y_{1}, \ldots, Y_{\mathrm{N}}\right\}$ simultaneous eigenstates $\left|y_{1}, \ldots, y_{N}\right\rangle$ with $y_{n}$ being the $Y_{n}$-eigenvalues, the generic $\mathrm{T}(\lambda)$-eigenstate $|t\rangle$ with eigenvalue $t(\lambda)$ is characterized by the following separate equations:

$$
F_{n}\left(y_{n}, \frac{i}{2 \pi} \frac{\partial}{\partial y_{n}}, t\left(y_{n}\right)\right) \Psi_{t}\left(y_{1}, \ldots ., y_{N}\right)=0, \quad \text { where } \Psi_{t}\left(y_{1}, \ldots ., y_{N}\right)=\left\langle y_{1}, \ldots ., y_{N} \mid t\right\rangle,
$$

$\forall n \in\{1, \ldots, \mathrm{N}\}$. Then it is natural searching for wave function solutions of factorized form $\Psi_{t}\left(y_{1}, \ldots, y_{N}\right)=\prod_{n=1}^{\mathrm{N}} Q_{t}^{(n)}\left(y_{n}\right)$, where $Q_{t}^{(n)}\left(y_{n}\right)$ is a solution of the equations (5) for the fixed $n \in\{1, \ldots, \mathbf{N}\}$. One of the fundamental contributions of Sklyanin ${ }^{13}$ has been to define a procedure to determine the quantum separate variables in the framework of QISM for the transfer matrix spectral problem and the explicit form of the corresponding quantum separate relations. In the class of integrable quantum models defined by a monodromy matrix $\mathrm{M}(\lambda)$ of the form (2) this procedure reads:

Sklyanin's procedure to SOV If the generator $\mathrm{B}(\lambda)$ of the Yang-Baxter algebra defines a one parameter family of simultaneously diagonalizable commuting operators with simple spectrum then the operator zeros $\left\{Y_{1}, \ldots, Y_{\mathrm{N}}\right\}$ of $\mathrm{B}(\lambda)$ define the quantum separate variables for the transfer matrix spectral problem. Moreover, the corresponding N separate equations are Baxter like second order difference equations computed in the spectrum of each quantum separate variable.

\section{The sine-Gordon model}

\subsection{Classical model}

The classical sine-Gordon model can be characterized by the Hamiltonian density $\mathrm{H}_{S G} \equiv\left(\partial_{x} \phi\right)^{2}+\Pi^{2}+8 \pi \mu \cos 2 \beta \phi$, where the field $\phi(x, t)$ is defined for $(x, t) \in$ $[0, R] \otimes \mathbb{R}$ with periodic boundary conditions $\phi(x+R, t)=\phi(x, t)$. The dynamics of the model in the Hamiltonian formalism is defined in terms of $\phi(x, t), \Pi(x, t)$ with $\{\Pi(x, t), \phi(y, t)\}=2 \pi \delta(x-y)$. The classical integrability of the sine-Gordon

${ }^{\mathrm{b}}$ Note that an independent proof of the completeness of the above factorized ansatz is required. 
model is assured thanks to the representation of the equation of motion by a zerocurvature condition $\left[\partial_{t}-V(x, t ; \lambda), \partial_{x}-U(x, t ; \lambda)\right]=0$. Here, we have defined $U=\mathrm{k}_{1} \sigma_{1} \cos \beta \phi+\mathrm{k}_{2} \sigma_{2} \sin \beta \phi-\mathrm{k}_{3} \sigma_{3} \Pi, V=-\mathrm{k}_{2} \sigma_{1} \cos \beta \phi-\mathrm{k}_{1} \sigma_{2} \sin \beta \phi-\mathrm{k}_{3} \sigma_{3} \partial_{x} \phi$ and $\mathrm{k}_{1}=i \beta(\pi \mu)^{1 / 2}\left(\lambda-\lambda^{-1}\right), \mathrm{k}_{2}=i \beta(\pi \mu)^{1 / 2}\left(\lambda+\lambda^{-1}\right), \mathrm{k}_{3} \equiv i \beta / 2$ and the $\sigma_{a}$ are the standard Pauli matrices.

\subsection{Quantum lattice regularization}

In order to regularize the ultraviolet divergences that arise in the quantization of the model a lattice discretization can be introduced. The field variables are discretized according to the standard recipe $\phi_{n} \equiv \phi(n \Delta)$ and $\Pi_{n} \equiv \Delta \Pi(n \Delta)$, where $\Delta=R / N$ is the lattice spacing. Then, the canonical quantization is defined by imposing that $\phi_{n}$ and $\Pi_{n}$ are self-adjoint operators satisfying the commutation relations $\left[\phi_{n}, \Pi_{m}\right]=2 \pi i \delta_{n, m}$. The quantum lattice regularization of the sine-Gordon model here used goes back to ${ }^{3}$ and it is characterized by the following Lax operator:

$$
\mathrm{L}_{n}(\lambda)=\kappa_{n}\left(\begin{array}{cc}
\mathrm{u}_{n}\left(q^{-1 / 2} \mathrm{v}_{n} \kappa_{n}+q^{1 / 2} \mathrm{v}_{n}^{-1} \kappa_{n}^{-1}\right) & \left(\lambda_{n} \mathrm{v}_{n}-\left(\mathrm{v}_{n} \lambda_{n}\right)^{-1}\right) / i \\
\left(\lambda_{n} / \mathrm{v}_{n}-\mathrm{v}_{n} / \lambda_{n}\right) / i & \mathrm{u}_{n}^{-1}\left(q^{1 / 2} \mathrm{v}_{n} \kappa_{n}^{-1}+q^{-1 / 2} \mathrm{v}_{n}^{-1} \kappa_{n}\right)
\end{array}\right)
$$

where $\lambda_{n} \equiv \lambda / \xi_{n}$ for any $n \in\{1, \ldots, \mathrm{N}\}$ and $\xi_{n}$ and $\kappa_{n}$ are the parameters of the model. Here, the basic operators are the unitary operators $\mathrm{v}_{n} \equiv e^{-i \beta \phi_{n}}$ and $\mathbf{u}_{n} \equiv e^{i \beta \Pi_{n} / 2}$ which generate $\mathbf{N}$ independent local Weyl algebras $\mathbf{u}_{n} \mathbf{v}_{m}=q^{\delta_{n m}} \mathbf{v}_{m} \mathbf{u}_{n}$, with parameter $q \equiv e^{-i \pi \beta^{2}}$, thanks to $\left[\phi_{n}, \Pi_{m}\right]=2 \pi i \delta_{n, m}$. Then the monodromy matrix that characterize the lattice sine-Gordon model is $M(\lambda) \equiv L_{N}(\lambda) \cdots L_{1}(\lambda)$ and it satisfies the Yang-Baxter equation w.r.t. the standard 6 -vertex $R$-matrix.

\subsection{Cyclic representations}

Here, we restrict our attention to the case in which $q$ is a $p$-root of unity, e.g. $\beta^{2}=p^{\prime} / p$ with $p$ odd and $p^{\prime}$ even coprime. This implies that the powers $p$ of the generators $\mathrm{u}_{n}$ and $\mathrm{v}_{n}$ are central elements of each local Weyl algebra. In this case, we can associate a $p$-dimensional linear space $\mathcal{H}_{n}$ to any site $n$ of the lattice and we can define on it the following cyclic representation of the Weyl algebra:

$$
\mathrm{v}_{n}\left|k_{n}\right\rangle=q^{k_{n}}\left|k_{n}\right\rangle, \quad \mathrm{u}_{n}\left|k_{n}\right\rangle=\left|k_{n}-1\right\rangle, \quad\left|k_{n}+p\right\rangle=\left|k_{n}\right\rangle, \forall k_{n} \in\{0, \ldots, p-1\} .
$$

Then, the quantum space associated to the lattice sine-Gordon model is the $p^{\mathrm{N}_{-}}$ dimensional Hilbert space $\mathcal{H} \equiv \otimes_{n=1}^{\mathrm{N}} \mathcal{H}_{n}$. In these representations the following definition $\mathcal{O}\left(\lambda^{p}\right) \equiv \prod_{k=1}^{p} \mathrm{O}\left(q^{k} \lambda\right)$ of average of a one-parameter family of commuting operators $\mathrm{O}(\lambda)$ plays a very important role; indeed it holds:

Proposition $2.1\left(^{\mathbf{1 4}}\right)$. The average of the monodromy matrix elements are central in the Yang-Baxter algebra and are characterized by:

$$
\begin{aligned}
& \mathcal{B}(\Lambda)=\mathcal{C}(\Lambda)=(F(-\Lambda)-F(\Lambda)) / 2, \quad \mathcal{A}(\Lambda)=\mathcal{D}(\Lambda)=(F(-\Lambda)+F(\Lambda)) / 2 \\
& F(\Lambda) \equiv \prod_{r=1}^{N}\left(\kappa_{r} \xi_{r} / i\right)^{p}\left(1+(-1)^{p^{\prime} / 2} i^{p}\left(\kappa_{r} / \xi_{r}\right)^{p} \Lambda\right)\left(1+(-1)^{p^{\prime} / 2} i^{p} \Lambda /\left(\kappa_{r} \xi_{r}\right)^{p}\right) / \Lambda
\end{aligned}
$$




\section{Transfer matrix spectral problem solution in SOV}

In ${ }^{14}$ the spectrum of the lattice sine-Gordon model has been completely characterized by SOV, we briefly summarize the results which mainly define the step A1 in the schema defined in subsection 1.2. In order to simplify the notations we will describe here and in the following only the case odd $\mathrm{N}$.

\subsection{Implementation of Sklyanin's SOV in sine-Gordon model}

In ${ }^{14}$ the recursive construction of the spectrum (eigenvalue and eigenstates) of the $\mathrm{B}(\lambda)$ Yang-Baxter generator has been implemented and the main results are here reproduced:

Proposition $\left.3.1 \mathbf{( 1 4}^{\mathbf{1 4}}\right)$. The operator zeros $\left\{Y_{1}, \ldots, Y_{\mathrm{N}}\right\}$ of $\mathrm{B}(\lambda)$ are simultaneously diagonalizable and with simple spectrum almost for all the values of the parameters of the Lax operators. Then, they define proper Sklyanin's quantum separate variables for the sine-Gordon spectral problem. Moreover, due to the centrality in the YangBaxter algebra of $\left\{Y_{1}^{p}, \ldots, Y_{\mathrm{N}}^{p}\right\}$, their spectrum is completely characterized by the identities $Y_{n}^{p}=Z_{n} \in \mathbb{C} \quad \forall n \in\{1, \ldots, \mathrm{N}\}$. The $Z_{n}$ are the zeros of the known Laurent polynomial $\mathcal{B}(\Lambda)$ defined in (8) while the decomposition of the identity in the $S O V$ basis is fixed by the following covectors-vector actions ${ }^{16}$ :

$$
\left\langle y_{1}^{\left(k_{1}\right)}, \ldots, y_{\mathrm{N}}^{\left(k_{\mathrm{N}}\right)} \mid y_{1}^{\left(h_{1}\right)}, \ldots, y_{\mathrm{N}}^{\left(h_{\mathrm{N}}\right)}\right\rangle=\prod_{n=1}^{\mathrm{N}} \delta_{k_{n}, h_{n}} \prod_{1 \leq b<a \leq \mathrm{N}}\left(y_{a}^{\left(h_{a}\right)} / y_{b}^{\left(h_{b}\right)}-y_{b}^{\left(h_{b}\right)} / y_{a}^{\left(h_{a}\right)}\right)^{-1}
$$

$\forall k_{n}, h_{n} \in\{0, \ldots, p-1\}$, where $y_{n}^{\left(k_{n}\right)} \equiv y_{n}^{(0)} q^{k_{n}}$ and $y_{n}^{(0)}$ is a fixed p-root of $Z_{n}$.

\subsection{Complete transfer matrix spectrum characterization}

Let us define the Laurent polynomials in $\lambda, d(\lambda)=q^{\mathrm{N}} a(-\lambda q)$ and $a(\lambda) \equiv$ $\prod_{r=1}^{N}\left(\kappa_{r} \xi_{r} / i \lambda\right)\left(1+i q^{-\frac{1}{2}} \lambda \kappa_{r} / \xi_{r}\right)\left(1+i q^{-\frac{1}{2}} \lambda / \kappa_{r} \xi_{r}\right)$, then it holds:

Proposition $3.2 \mathbf{( 1 4}^{\mathbf{1 4}}$. The spectrum of the transfer matrix $\mathrm{T}(\lambda)$ is simple and the set $\Sigma_{\mathrm{T}}$ of the eigenvalues functions coincides with the set of $t(\lambda)$ solutions of the Baxter equation:

$$
t(\lambda) Q_{t}(\lambda)=a(\lambda) Q_{t}\left(\lambda q^{-1}\right)+d(\lambda) Q_{t}(\lambda q), \quad \forall \lambda \in \mathbb{C},
$$

in the class of functions $\lambda^{(\mathrm{N}-1)} t(\lambda) \in \mathbb{R}\left[\lambda^{2}\right]_{\mathrm{N}-1}, Q_{t}(\lambda) \in \mathbb{R}[\lambda]_{\mathrm{N}(p-1)}$, where $\mathbb{R}[\lambda]_{M}$ is the linear space of real polynomials of degree $\leq M$ in $\lambda$. Moreover, the unique (up to normalization) eigenstate $|t\rangle$ corresponding to $t(\lambda) \in \Sigma_{\mathbf{T}}$ is characterized by ${ }^{14,16}$ :

$$
|t\rangle=\sum_{h_{1}, \ldots, h_{\mathrm{N}}=1}^{p} \prod_{a=1}^{\mathrm{N}} Q_{t}\left(y_{a}^{\left(h_{a}\right)}\right) \prod_{1 \leq b<a \leq \mathrm{N}}\left(y_{a}^{\left(h_{a}\right)} / y_{b}^{\left(h_{b}\right)}-y_{b}^{\left(h_{b}\right)} / y_{a}^{\left(h_{a}\right)}\right)\left|y_{1}^{\left(h_{1}\right)}, \ldots, y_{\mathrm{N}}^{\left(h_{\mathrm{N}}\right)}\right\rangle .
$$




\section{Matrix elements of local operators}

In ${ }^{16}$ the steps B1, B2 and B3 of the schema defined in subsection 1.2 have been derived, we briefly summarize the final results on matrix elements:

Proposition $4.1\left({ }^{\mathbf{1 6}}\right)$. There exists a basis $\mathbb{B}_{\mathcal{H}}$ in $\operatorname{End}(\mathcal{H})$ such that for any $\mathrm{O} \in$ $\mathbb{B}_{\mathcal{H}}$ the matrix elements on the transfer matrix eigenstates read:

$\left\langle t^{\prime}|\mathrm{O}| t\right\rangle=\operatorname{det}_{\mathrm{N}}\left\|\Phi_{a, b}^{\left(\mathrm{O}, t^{\prime}, t\right)}\right\|, \quad \Phi_{a, b}^{\left(\mathrm{O}, t^{\prime}, t\right)} \equiv\left(y_{a}^{(0)}\right)^{2 b-1} \sum_{c=1}^{p} F_{\mathrm{O}, b}\left(y_{a}^{(c)}\right) Q_{t}\left(y_{a}^{(c)}\right) Q_{t^{\prime}}\left(-y_{a}^{(c)}\right) q^{(2 b-1) c}$,

where the coefficients $F_{\mathrm{O}, b}\left(y_{a}^{(c)}\right)$ characterize the operator $\mathrm{O}$ and are computed by using the solution of the quantum inverse problem. Let us show two examples:

a) If $\mathrm{O}$ is the identity operator, it holds $F_{\mathbf{u}_{1}, b}\left(y_{a}^{(c)}\right)=1$ for any $a, b \in\{1, \ldots, \mathbf{N}\}$.

b) If $\mathrm{O} \equiv \mathrm{u}_{1}$ is the Weyl algebra local generator in site 1, it holds:

$$
\begin{aligned}
& F_{\mathrm{u}_{1}, b}\left(y_{a}^{(c)}\right)=y_{a}^{(c)} \quad \forall b \in\{1, \ldots, \mathrm{N}-1\}, \forall a \in\{1, \ldots, \mathrm{N}\} \\
& F_{\mathrm{u}_{1}, \mathrm{~N}}\left(y_{a}^{(c)}\right)=\frac{\left(y_{a}^{(0)}\right)^{2(\mathrm{~N}-1)} q^{1 / 2} \xi_{1} q^{(c+1)(\mathrm{N}-1)} Q_{t}\left(y_{a}^{(c)}\right) Q_{t^{\prime}}\left(-y_{a}^{(c+1)}\right)}{\prod_{n=2}^{\mathrm{N}} \kappa_{n} / i\left(q\left(\xi_{1} \kappa_{1}\right)^{2}+\left(y_{a}^{(c+1)}\right)^{2}\right) Q_{t^{\prime}}\left(-y_{a}^{(c)}\right)} a\left(\eta_{a}^{(c+1)}\right) .
\end{aligned}
$$

\section{Conclusion and outlook}

Let us complete this contribution evidencing the fundamental feature of universality which emerges in the characterization of both the spectrum and the dynamics by our approach in SOV. Indeed, this appears clearly by the analysis of several others fundamental integrable quantum models associated to more general cyclic representations, to highest weight representations of 6-vertex and dynamical 6-vertex as well as to spin-1/2 representations of 8-vertex Yang-Baxter algebra and of general 6vertex reflection algebra. Indeed, the results derived in ${ }^{14-16}$ show that a part from model dependent features, like the nature of the spectrum of the quantum separate variables, the coefficients in the Baxter equation and the SOV-reconstruction of local operators, the spectrum and dynamics admit the same type of representations. In particular, the form factors are expressible as determinants of matrices with elements the "convolutions" over the spectrum of the separate variables of Baxter equation solutions plus contributions coming from the local operators; i.e. (13) seems universal. The next natural step is to complete for the sine-Gordon model the described integrable microscopic program. Indeed, the main point to complete is B4 which defines the form factors in the infinite volume limit and allows for the comparison with those in the S-matrix formulation in this way solving the local field identification problem. Finally, the most intriguing projects are related to the generalization of this analysis to more advanced quantum models like those associated to higher rank quantum spin chains of fundamental interest in gauge theories and also to models like Hubbard model which is a celebrated model in condensed matter theory as it describes both the charge and the spin degrees of freedom.

\section{Acknowledgments}

The author would like to gratefully acknowledge the fundamental contributions given by J. Teschner in ${ }^{14}$ and N. Grosjean and M.-J. Maillet in ${ }^{16}$ to the subjects here described. I gratefully acknowledge the YITP Institute of Stony Brook for the opportunity to develop 
my research programs under support of National Science Foundation grants PHY-0969739.

\section{References}

1. W. Heisenberg, Z. Phys. 49 (1928) 619; H. Bethe, Z. Phys. 71 (1931) 205; E. Lieb and D. Mattis, Mathematical Physics in One Dimension, New York: Academic Press, 1966.

2. C. N. Yang, Phys. Rev. Lett. 19 (1967) 1312; L. D. Faddeev and L. A. Takhtajan, Russ. Math. Surveys, 34 : 5 (1979) 11; V. E. Korepin, N. M. Bogoliubov, A. G. Izergin, Quantum Inverse Scattering Method and Correlation Functions, Cambridge: Cambridge Univ. Press, 1993; M. Jimbo and T. Miwa, Algebraic analysis of solvable lattice models, AMS, 1995.

3. L. D. Faddeev, E. K. Sklyanin and L. A. Takhtajan, Theor. Math. Phys. 40 (1980) 688; A.G. Izergin, V.E. Korepin, Nucl. Phys. B 205 (1982) 401; V. O. Tarasov, I. A. Takhtadzhyan and L. D. Faddeev, Theor. Math. Phys. 57, 2 (1983) 1059.

4. A. B. Zamolodchikov, JEPT Lett. 25 (1977) 468; A. B. Zamolochikov, Al. B. Zamolodhikov, Nucl. Phys. B 133 (1978) 525, Moscow preprint ITE P- 112 (1977); Ann. Phys. 120 (1979) 253; M. Karowski, H.J. Thun, T.T. Truong and P. Weisz, Phys. Lett. B 67 (1977) 321; M. Karowski, H.J. Thun, Nucl. Phys. B 130 (1977) 295; B. Berg, M. Karowski, V. Kurak and P. Weisz, Nucl. Phys. B 134 (1978) 125; M. Karowski, Phys. Rep. 49 (1979) 229.

5. M. Karowski and P. Weisz, Nucl. Phys. B 139 (1978) 445; B. Berg, M. Karowski and P. Weisz, Phys. Rev. D 19 (1979) 2477; F. A. Smirnov, J. Phys. A 17 (1984) L873; J. Phys. A 19 (1986) L575; Form factors in completely integrable models of quantum field theory, Adv. Series in Math. Phys. 14, World Scientific, 1992.

6. A. B. Zamolodchikov, Adv. Stud. Pure Math. 19 (1989) 641; J. Cardy, Phys. Rev. Lett. 60 (1988), 2709; F. A. Smirnov, Int. J. Mod. Phys. A 4 (1989) 4213; N.Yu. Reshetikhin and F. A. Smirnov, Comm. Math. Phys. 131 (1990) 157; F. A. Smirnov, Nucl. Phys. B 337 (1990) 156; Al.B. Zamolodchikov, Nucl. Phys. B 348 (1991) 619.

7. J. Cardy and G. Mussardo, Nucl. Phys. B 340 (1990) 387; A. Koubek, Nucl. Phys. B 435 (1995) 703; F. Smirnov, Nucl. Phys. B 453 (1995) 807; M. Jimbo, T. Miwa, Y. Takeyama, arXiv:math-ph/0303059

8. G. Delfino and G. Niccoli, Nucl. Phys. B707(2005) 381; J. Stat. Mech. (2005) P04004; JHEP 05 (2006) 035; Nucl. Phys. B 799 [FS] (2008) 364.

9. O. Babelon, D. Bernard, F. A. Smirnov, Comm. Math. Phys. 182 (1996) 319; Comm. Math. Phys. 186 (1997) 601.

10. H. Boos, M. Jimbo, T. Miwa, F. Smirnov, and Y. Takeyama, Com. Math. Phys. 272 (2007) 263; arXiv:hep-th/0702086 Comm. Math. Phys. 286 (2009) 875; M. Jimbo, T. Miwa, F. Smirnov, arXiv:0912.0934 J. Phys. A 42 (2009) 304018; arXiv:1103.1534 Lett. Math. Phys. 96 (2011) 325; H. Boos, M. Jimbo, T. Miwa, F. Smirnov, arXiv:0911.3731.

11. M. Jimbo, T. Miwa, F. Smirnov, Nucl. Phys. B 852 (2011) 390.

12. N. Kitanine, J. M. Maillet and V. Terras, Nucl. Phys. B 554 (1999) 647; J. M. Maillet and V. Terras, Nucl. Phys. B 575 (2000) 627; N. Kitanine, J. Phys. A 34 (2001) 8151; O. A. Castro-Alvaredo, J. M. Maillet, J. Phys. A 40 (2007) 7451; N. Kitanine, K. K. Kozlowski, J. M. Maillet, G. Niccoli, N. A. Slavnov, V. Terras, J. Stat. Mech. (2007) P10009; J. Stat. Mech. (2008) P07010.

13. E. K. Sklyanin, Lect. Notes Phys. 226 (1985) 196; Quantum inverse scattering method. Selected topics, in: Quantum groups and quantum integrable systems, World Scientific, 1992; Prog. Theor. Phys. Suppl. 118 (1995) 35.

14. G. Niccoli and J. Teschner, J. Stat. Mech. (2010) P09014.

15. G. Niccoli, Nucl. Phys. B 835 (2010) 263 ; JHEP 03 (2011) 123 ; arXiv:1205.4537; J. Stat. Mech. (2012) P10025 ; arXiv:1206.2418; arXiv:1207.1928; N. Grosjean and G. Niccoli, J. Stat. Mech. (2012) P11005.

16. N. Grosjean, J. M. Maillet, G. Niccoli, J. Stat. Mech. (2012) P10006. 